\title{
Tapered Optical Fiber-Based LSPR Biosensor for Ascorbic Acid Detection
}

\author{
Guo ZHU ${ }^{1}$, Lokendra SINGH ${ }^{1,2}$, Yu WANG ${ }^{1}$, Ragini SINGH ${ }^{3}$, Bingyuan ZHANG ${ }^{1 *}$, \\ Fengzhen LIU ${ }^{4}$, Brajesh Kumar KAUSHIK ${ }^{5,1}$, and Santosh KUMAR ${ }^{1,5,6^{*}}$ \\ ${ }^{1}$ Shandong Key Laboratory of Optical Communication Science and Technology, School of Physics Science and \\ Information Technology, Liaocheng University, Liaocheng 252059, China \\ ${ }^{2}$ Department of Robotics and Automation Engineering, PARK College of Engineering and Technology, Kaniyur 641659, \\ India \\ ${ }^{3}$ School of Agriculture Science, Liaocheng University, Liaocheng 252059, China \\ ${ }^{4}$ Liaocheng People's Hospital, Medical College of Liaocheng University, Liaocheng 252000, China \\ ${ }^{5}$ Department of Electronics and Communication Engineering, Indian Institute of Technology-Roorkee, Roorkee 247667, \\ India \\ ${ }^{6}$ Department of Electrical and Electronics \& Communication Engineering, DIT University, Dehradun 248009, India \\ *Corresponding authors: Bingyuan ZHANGandSantosh KUMAR E-mail: zhangbingyuan@lcu.edu.cnandsantoshrus@yahoo.com
}

\begin{abstract}
The ascorbic acid (AA) is a biomarker that can be used to detect the symptoms of severe disorders such as scurvy, Parkinson's, Alzheimer's, and cardiovascular diseases. In this work, a simple and effective sensor model is developed to diagnose the presence of AA samples. To develop the sensor, a tapered single-mode optical fiber has been used with the well-known phenomenon of localized surface plasmon resonance (LSPR). For LSPR, the tapered region is immobilized with synthesized gold nanoparticles (AuNPs) and zinc oxide nanoparticles (ZnO-NPs) whose absorbance peak wavelengths appear at $519 \mathrm{~nm}$ and $370 \mathrm{~nm}$, respectively. On the basis of nanoparticles (NPs) configurations, two different biosensor probes are developed. In the first one, the sensing region is immobilized with AuNPs and named Probe I. In the second probe, the immobilized layer of AuNPs is further coated with a layer of ZnO-NPs, and a resultant probe is termed as Probe II. The characterizations of synthesized AuNPs and developed fiber probes are done by the ultraviolet-visible (UV-vis) spectrophotometer, high-resolution transmission electron microscope (HR-TEM), atomic force microscopy (AFM), and scanning electron microscope (SEM). To enhance the selectivity, a sensing region of probes is functionalized with ascorbate oxidase enzyme that oxidizes the AA in the presence of oxygen. The response of developed sensor probes is authenticated by sensing the samples of AA in the range from $500 \mathrm{nM}$ to $1 \mathrm{mM}$, which covers the range of AA found in human bodies, i.e., $40 \mu \mathrm{M}-120 \mu \mathrm{M}$. The performance analysis of the developed sensor probes has been done in terms of their stability, reproducibility, reusability, and selectivity. To observe the stability of AA, a pH-test has also been done that results in a better solubility of AA molecules in phosphate-buffered saline (PBS) solution.
\end{abstract}

Keywords: Ascorbic acid; localized surface plasmon resonance; gold nanoparticles; zinc oxide nanoparticles; tapered fiber; optical fiber sensor

Citation: Guo ZHU, Lokendra SINGH, Yu WANG, Ragini SINGH, Bingyuan ZHANG, Fengzhen LIU, et al., "Tapered Optical Fiber-Based LSPR Biosensor for Ascorbic Acid Detection," Photonic Sensors, 2021, 11(4): 418-434.

Received: 30 December 2019 / Revised: 20 August 2020

(C) The Author(s) 2020. This article is published with open access at Springerlink.com

DOI: $10.1007 / \mathrm{s} 13320-020-0605-2$

Article type: Regular 


\section{Introduction}

The chemical oxidation is one of the most common processes within the human bodies that leads to the generation of various radicals and singlets [1]. Antioxidants, such as ascorbic acid (AA), also known as vitamin $\mathrm{C}$, and inhibit oxidation are responsible for the proper functioning of living organisms [2]. The recommended necessary amount of AA is $100 \mathrm{mg}$ to $200 \mathrm{mg}$ per day [3]. The healthy human bodies have the presence of AA approximately in the range of $40 \mu \mathrm{M}$ $-120 \mu \mathrm{M}$ [4]. The presence of AA was claimed several decades ago in various foods and drinks and has been continuously diagnosed for the evaluation of its sources. The malnutrition of AA in the human body is closely related to severe symptoms such as scurvy, Parkinson's, Alzheimer's, and cardiovascular diseases $[5,6]$. Therefore, for the detection of AA, several methods have been proposed including electrochemistry [7, 8], fluorescence [9, 10], calorimetry [11], liquid chromatography [12], and ultraviolet-visible (UV-vis) spectrometry [13]. However, these methods are not very attractive because of their low sensitivity, limited selectivity, and complicated procedures. To overcome the limitations of the aforementioned techniques, an optical fiber technology has been widely used for the development of optical biosensors [14]. To develop the optical fiber biosensors, several techniques are employed such as core mismatching [15], tapering [16-19], and gratings [20]. Among all the techniques, tapering of the optical fiber attracts a lot of attractions due to its easy fabrication and higher sensitivity [21]. For instance, a tapered optical fiber structure with a diameter of $1 \mu \mathrm{m}$ was used to sense the presence of cancer biomarkers with very fine sensitivity [17]. In another structure, a tapered optical fiber structure was used to sense the presence of glucose solutions in real samples, and the attained results were given with a better sensitivity and a high autocorrelation [18]. A tapered fiber structure with a diameter of $5 \mu \mathrm{m}$ was used with gold nanoclusters as a metallic layer to diagnose the availability of biomolecules with a very high linearity range of $99.30 \%$ [19]. Recently, metal nanoparticles (MNPs) based on tapered optical fiber structures have been widely used for the detection of biomarkers [22]. Therefore, in this work, gold nanoparticles (AuNPs) and zinc oxide nanoparticles (ZnO-NPs) immobilized tapered fiber structures (TFSs) are used to detect the presence of AA samples. On the basis of different combinations of NPs, two different probes are developed. In one probe, a uniform layer of AuNPs is immobilized over the tapered region and termed as Probe I. In another one, a layer of $\mathrm{ZnO}-\mathrm{NPs}$ is immobilized over the AuNPs layer and the probe is coined as Probe II. Moreover, to reduce the effect of other biomarkers and increase the selectivity towards AA, the NPs immobilized probes are functionalized by ascorbate oxidase (AOx) enzyme. To authenticate the response of developed sensor probes, AA samples are prepared in the range from $500 \mathrm{nM}$ to $1 \mathrm{mM}$ and tested through both the developed sensor probes. For the validation of the solubility of AA molecules, several $\mathrm{pH}$ value solutions are prepared and tested through the developed probes. The complete manuscript is comprised of four major sections. Section 1 presents a brief introduction to the importance of AA and its detection through existing technologies. To develop the sensor probes, the used material and methods are discussed in Section 2. Thereafter, the results obtained for the validation of MNPs solutions and developed sensor probes are discussed in Section 3 and this section also includes the sensing performance results of the developed sensor probes. Finally, the findings of the work are concluded in Section 4.

\section{Materials and methods}

\subsection{Materials}

Chloroauric acid $\left(\mathrm{HAuCl}_{4}\right)$, sodium citrate 
$\left(\mathrm{C}_{6} \mathrm{H}_{5} \mathrm{Na}_{3} \mathrm{O}_{7}\right)$, and deionized (DI) water are used for the synthesis of AuNPs. Zinc Oxide ( $\mathrm{ZnO})$ NPs (Product no. 721077, Sigma-Aldrich) is used for immobilizing the probes with the $\mathrm{ZnO}-\mathrm{NPs}$ layer. Acetone, hydrogen peroxide $\left(\mathrm{H}_{2} \mathrm{O}_{2}\right)$ solution, sulphuric acid $\left(\mathrm{H}_{2} \mathrm{SO}_{4}\right)$ solution, ethanol, and nitrogen $\left(\mathrm{N}_{2}\right)$ gas are used for the cleaning of fiber probes. (3-mercaptopropyl) trimethoxysilane (MPTMS), 11-mercaptoundecanoic acid (MUA), $N$-(3-dimethylaminopropyl)- $N$ '-ethylcarbodiimide hydrochloride (EDC), $N$-hydroxysuccinimide (NHS), phosphate-buffered saline (PBS), and ascorbate oxidase from cucurbita species (Product No. A0157, Sigma-Aldrich) are used for functionalizing the probes with a layer of NPs and enzyme. Sodium hydroxide $(\mathrm{NaOH})$ is used to maintain the $\mathrm{pH}$. Almost all the reagents are of analytical grade and purchased from Sigma-Aldrich, Shanghai, China. Some common chemicals such as ethanol, nitric acid $\left(\mathrm{HNO}_{3}\right)$, hydrochloric acid $(\mathrm{HCl})$, sulfuric acid $\left(\mathrm{H}_{2} \mathrm{SO}_{4}\right)$, acetone $\left(\mathrm{C}_{3} \mathrm{H}_{6} \mathrm{O}\right)$, ascorbic acid, glucose, urea, D-galactose, and dopamine are purchased from a local vendor available at Liaocheng, Shandong, China.

\subsection{Fabrication and sensing mechanism of the tapered optical fiber structure}

To fabricate the TFSs, a single-mode fiber (SMF) is used with an initial core and cladding diameters of $9 \mu \mathrm{m}$ and $125 \mu \mathrm{m}$, respectively, through the advanced anaerobic plasma tapering technology-based 3SAE combiner manufacturer system (CMS) (3SAE CMS Technologies, Inc., USA machine). The tapering of the optical fiber is done to increase the outflow of evanescent waves (EWs) [23]. The schematic of TFSs is shown in Fig. 1, where the length and diameter of the sensing region are $4 \mathrm{~mm}$ and $40 \mu \mathrm{m}$, respectively. The developed sensor probes work on the principle of the localized surface plasmon resonance (LSPR) phenomenon that results from the combination of the surface plasmon wave (SPW) and exponentially decaying EWs [24]. In a normal region of the fiber, the optical signal obeys the principle of total internal reflection (TIR), whereas, in the tapered region, the power of the signal is exponentially decayed as shown in Fig. 1. The decaying EW penetrates into the cladding up to a few microns and the length is known as the penetration length which can be calculated as [25]

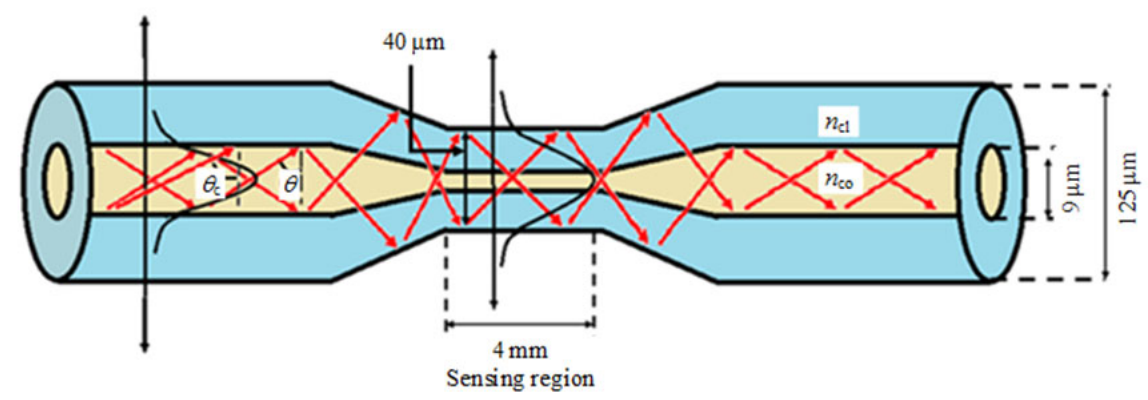

Fig. 1 Schematic of tapered optical fiber structure.

$$
d_{p}=\frac{\lambda}{2 \pi\left(n_{\mathrm{co}}^{2} \sin ^{2} \theta-n_{\mathrm{cl}}^{2}\right)^{1 / 2}}
$$

where $\theta$ is the incident angle of an optical signal at the interface between the core and cladding, and $n_{\mathrm{co}}$ and $n_{\mathrm{cl}}$ are refractive indices of the core and cladding, respectively. Optical fiber-based biosensors are working on the principle of the surface plasmon resonance (SPR) phenomenon. The
SPR phenomenon gets initiated at the interface of cladding and coated MNPs, due to the application of exponentially decaying EWs. The EWs jiggle the MNPs at a higher frequency and sets a wave termed as SPWs. When the phases between EWs and SPWs match, the phenomenon is termed as SPR. If the size of the used MNPs is in the range of the nanometer-scale, then SPWs get localized, and the resultant phenomenon is termed as LSPR [26]. On 
the application of a change in refractive index (RI) in the vicinity of the sensor head, the phase of interacting EWs gets changed and further alters the peak resonance wavelength. Therefore, in this work, an AA solution is used to change the RI of the sensor head and a shift in peak resonance wavelength is recorded for the performance analysis of the developed sensor probes.

\subsection{Synthesis of gold nanoparticles}

The synthesis of AuNPs is done by obeying the Turkevich protocol as discussed in [27]. In brief, $\mathrm{HAuCl}_{4}(150 \mu \mathrm{L}, 100 \mathrm{mM})$ is reduced under the influence of $1.8 \mathrm{ml}(38 \mathrm{mM})$ sodium citrate solution in $14.85 \mathrm{ml}$ of DI water. Firstly, the $\mathrm{HAuCl}_{4}$ is added in DI water and heated at $100{ }^{\circ} \mathrm{C}$ for 15 minutes. As the solution starts boiling, sodium citrate solution is added under constant stirring. After 5 minutes, the color of the solution turns into red wine color that primarily confirms the synthesis of AuNPs. Afterward, the stabilization of the solution is attained by continuing the stirring for the next 10 minutes.

\subsection{Coating of nanoparticles over the sensing region of fiber probes}

For the deposition of NPs over the sensing region, a dip coating method is employed. For accuracy, all-fiber probes are immobilized with NPs with the same solutions. Initially, the fiber probes are cleaned by using acetone to remove any organic ash and smoothen the cladding surface. Then, the cleaned sensing regions of probes are hydrolyzed by dipping them in the piranha solution $\left(3: 7 \mathrm{v} / \mathrm{v}\right.$ of $\mathrm{H}_{2} \mathrm{O}_{2}$ : $\mathrm{H}_{2} \mathrm{SO}_{4}$ ) for 30 minutes [28]. Thereafter, the fiber probes are thoroughly rinsed with DI water and dried in an oven at $70{ }^{\circ} \mathrm{C}$ for 20 minutes. After 4 hours, dried fiber probes are dipped in the ethanolic 1\% MPTMS solution for the next 12 hours. The MPTMS is a silane agent that is useful for the adhesion of AuNPs to the available $\mathrm{OH}$ groups on the fiber surface [29]. Afterwards, the unbound molecules of MPTMS are removed by rinsing the probes with ethanol and dried by nitrogen gas. Then, salinized fiber probes are dipped in freshly prepared AuNPs aqueous solutions [22].

After 48 hours, the unbound monomers of AuNPs are removed by rinsing the probes with ethanol and dried by using nitrogen gas. The resultant probes are termed as Probe I. In a similar manner, the probes termed as Probe II are fabricated by depositing a layer of ZnO-NPs over the AuNPs immobilized probes. The AuNPs immobilized probes are dipped in aqueous solutions of $\mathrm{ZnO}-\mathrm{NPs}$ (5\% wt.) for 10 minutes and annealed at $70{ }^{\circ} \mathrm{C}$ for 30 minutes [30]. The uniform deposition of the $\mathrm{ZnO}-\mathrm{NPs}$ layer is attained by repeating the process three times as shown in Fig. 2. The layer of $\mathrm{ZnO}-\mathrm{NPs}$ is deposited to enhance the RI of the sensing region [31].

\subsection{Characterization}

The synthesized AuNPs and ZnO-NPs solutions are first characterized by a UV-vis spectrophotometer, a high-resolution transmission electron microscope (HR-TEM), and an atomic force microscopy (AFM). The UV-vis spectrophotometer (HITACHI-U-3310) is used to record the absorbance spectrum. The distribution of NPs within the solution is determined by HR-TEM (Talos L120C, Thermo Fisher Scientific). The characterization of the NPs coated fiber probe is done by the scanning electron microscope (SEMGemini, Carl Zeiss microscopy).

\subsection{Functionalization of sensor probes I and II with enzyme}

The enzyme functionalization is done to increase the specific response of probes towards AA. Initially, the NPs coated probes are immersed in an ethanolic MUA solution $(10 \mathrm{ml}, 0.5 \mathrm{mM})$ for 5 hours. MUA immobilize a layer of the carboxylic group over the surface of NPs [32]. Afterwards, the activation of deposited carboxylic groups is done by treating the probes with the $5 \mathrm{ml}$ solution of EDC $(200 \mathrm{mM})$ and NHS $(50 \mathrm{mM})$. After 10 minutes, the fiber probes are 
dipped in an aqueous solution of the AOx solution for 12 hours. The esters groups of NHS are reacted with the AOx and forms a uniform coating of enzyme over the sensing surface as shown in Fig. 3 [33]. The stock solution of AOx enzyme
( 100 units $/ \mathrm{ml}$ ) is prepared by dissolving the AOx in $1 \mathrm{ml}$ of the PBS solution ( $\mathrm{pH} 7.4$ ). From a stock solution, $50 \mu 1$ of AOx enzyme in $4.95 \mathrm{ml}$ of the PBS solution is used for the functionalization of probes.
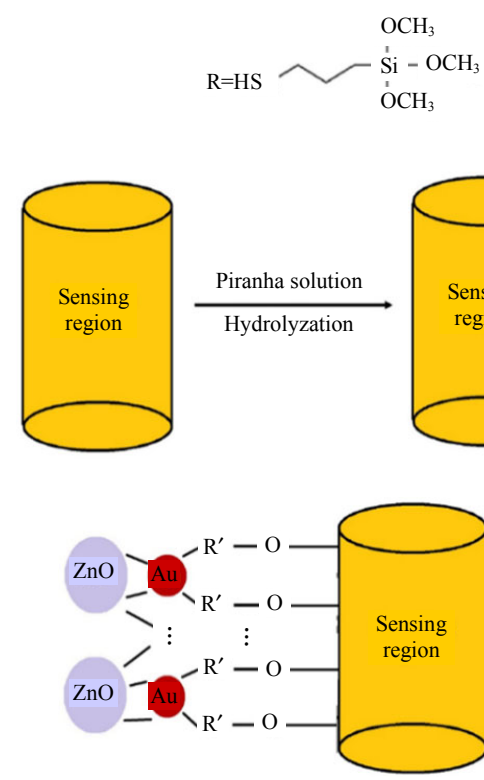
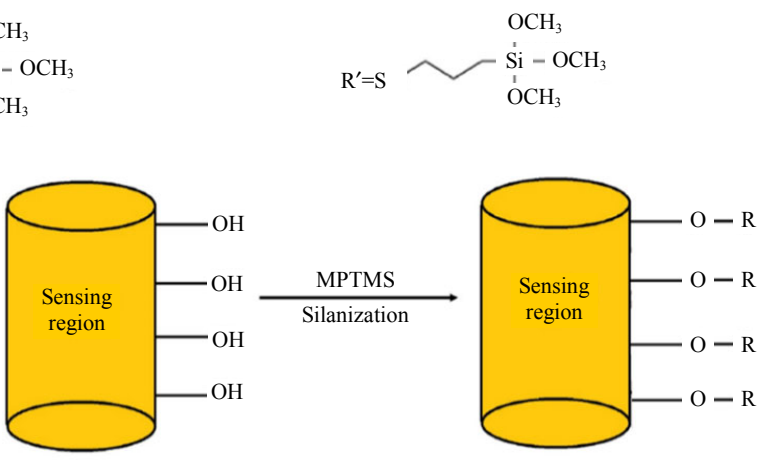

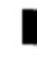

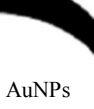

Fig. 2 Immobilization of nanoparticles over the sensing region of fiber probes.

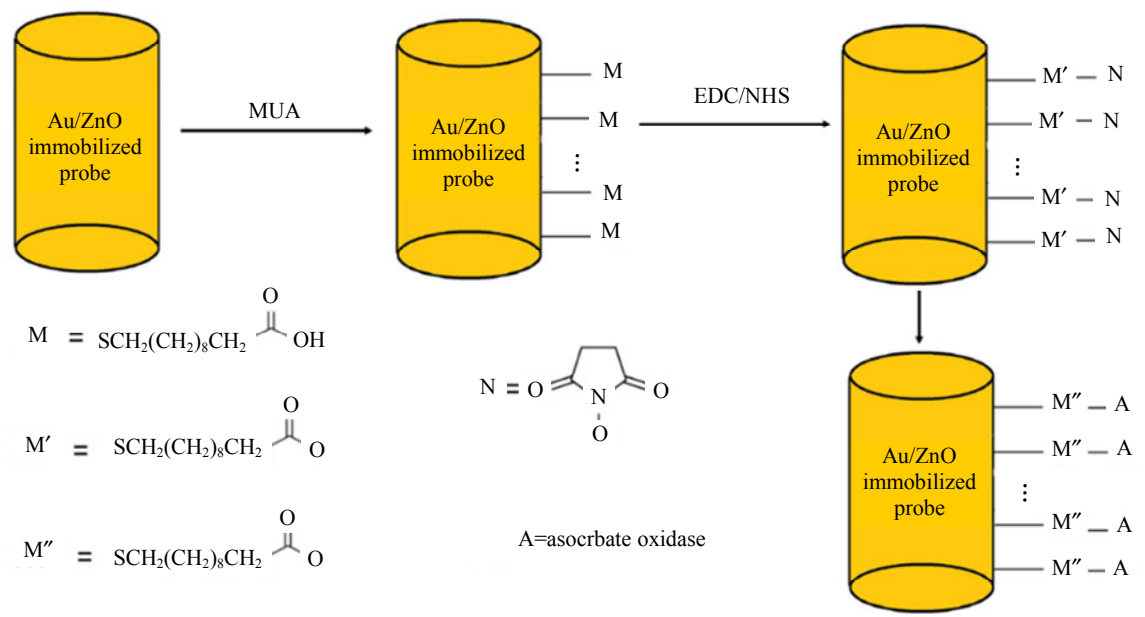

Fig. 3 Functionalization of enzyme over nanoparticles-coated fiber probes.

\subsection{Preparation of the ascorbic acid solution}

To characterize the sensing capabilities of AOx sensor probes, various concentrations of AA are prepared. The level of AA in the human body is in the range of $40 \mu \mathrm{M}-120 \mu \mathrm{M}$. Therefore, solutions of twelve different concentrations of AA are prepared which consist of the lowest and highest concentrations found in human bodies. Firstly, a stock solution of $10 \mathrm{mM}$ is prepared by dissolving the $0.0353 \mathrm{gm}$ of AA in the $20 \mathrm{ml} 1 \mathrm{X}$ PBS solution (pH 7.4). The AA samples are easily soluble in the PBS solution due to its favorable salinity and solvent properties [34].

Thereafter, the remaining lower concentrations, such as $500 \mathrm{nM}, 1 \mu \mathrm{M}, 10 \mu \mathrm{M}, 20 \mu \mathrm{M}, 50 \mu \mathrm{M}$, $100 \mu \mathrm{M}, 150 \mu \mathrm{M}, 200 \mu \mathrm{M}, 500 \mu \mathrm{M}$, and $1 \mathrm{mM}$, are prepared by diluting the stock solution in PBS. To 
prepare all the AA concentration solutions, the $1 \mathrm{X}$ PBS solution is used because $\mathrm{pH}$ (7.4) of the $1 \mathrm{X}$ PBS solution is equivalent to the $\mathrm{pH}$ of human $\operatorname{serum}(7.35-7.45)[35]$.

\subsection{Experimental setup for the detection of ascorbic acid}

The response of the developed sensor probes is observed by testing the wide concentration of AA solutions. The experimental setup is consisting of a halogen-lamp light source (HL-1000, Shanghai Wen Yi Photoelectric Technology Co., Ltd.) to propagate the light signals. Then, the transmitted signal is recorded through the HR2000 + spectrometer (Ocean Optics Inc., USA). In between the source and detector, a sensor probe is spliced by a fusion-splicer as shown in Fig. 4. The extended views of both the developed sensor probes are also shown in the inset of Fig. 4.

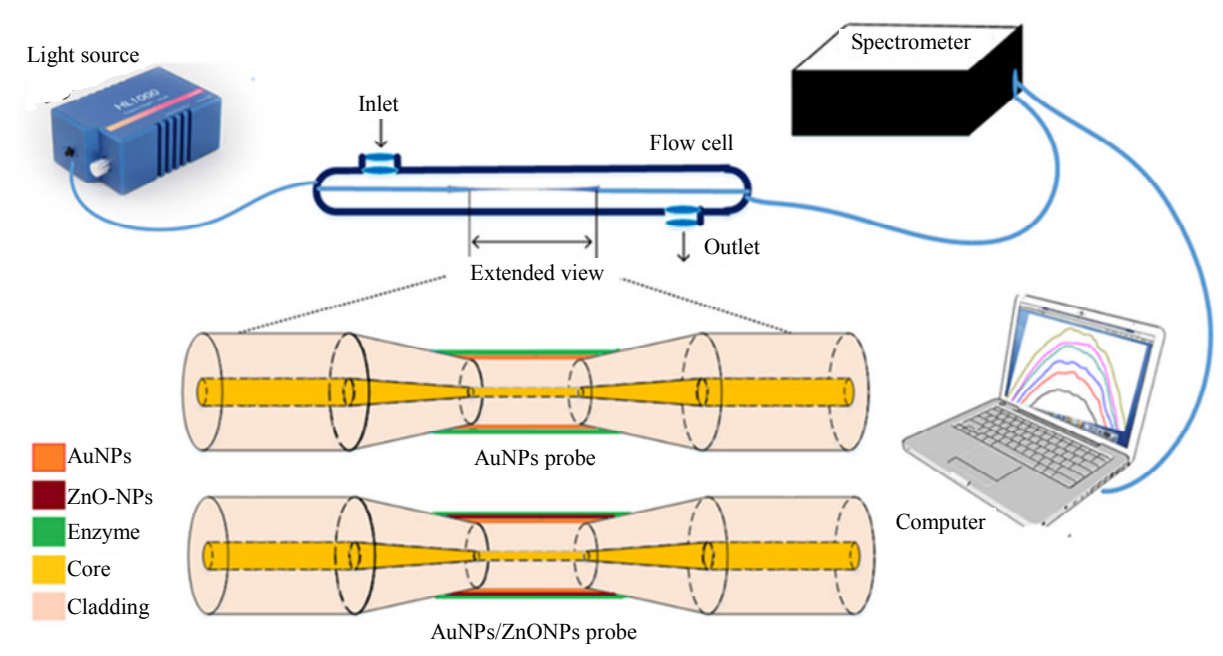

Fig. 4 Experimental setup for the sensing of ascorbic acid through the tapered fiber sensor probe.

\section{Results and discussion}

\subsection{Analysis of the tapered fiber diameter}

The sensitivity of the tapered fiber sensor mainly depends on the diameter of the tapered region. The analysis is done by fabricating four different diameters of tapered fibers such as $25 \mu \mathrm{m}, 40 \mu \mathrm{m}$, $50 \mu \mathrm{m}$, and $60 \mu \mathrm{m}$. The numerical values of the diameter of the fabricated fibers are saved and plotted by using MATLAB as shown in Fig. 5. Thereafter, the transmitted intensity spectrum is recorded through the tapered fiber and plotted together as shown in Fig. 6. From the results, it is conceived that the lowest intensity counts are obtained through the $25 \mu \mathrm{m}$ diameter tapered fiber. The lowest intensity count means the most of the power is flowing out from the tapering region that will enhance the sensitivity of the developed sensors. But, due to the high fragility of the $25 \mu \mathrm{m}$ diameter tapered fiber, it is quite difficult to complete the whole fabrication process. It is also ascertained that the intensity counts obtained through the $40 \mu \mathrm{m}$ diameter taper fiber is a bit higher than that of the $25 \mu \mathrm{m}$ diameter tapered fiber and also easy to fabricate the sensor structure. Therefore, for the proposed work, the tapered fiber with a diameter of $40 \mu \mathrm{m}$ is used to develop the proposed sensor probes. Further, to observe the homogeneity of the $40 \mu \mathrm{m}$ tapered fiber structure, four different tapered fibers are fabricated and analyzed. From the attained results, it can be concluded that the diameter of all tapered fiber structures sustains homogeneity as presented in Fig.7(a). The fabricated tapered fibers are analyzed in terms of the measurement of transmitted intensity, and the results are presented in Fig. 7(b). It can be concluded from these results that the fabricated fiber probes sustain homogeneity in terms of the diameter and peak wavelength. 


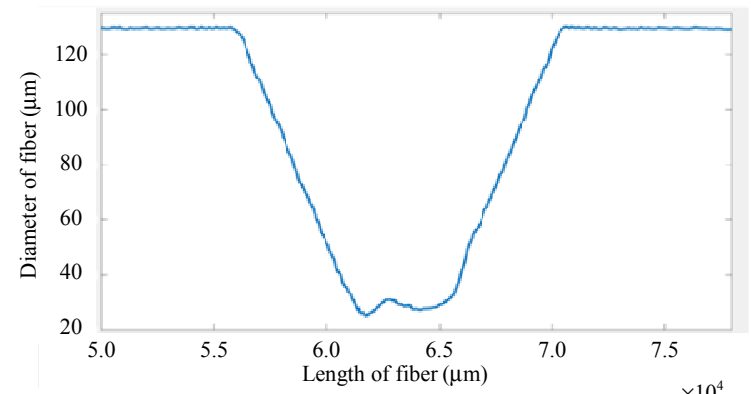

(a)

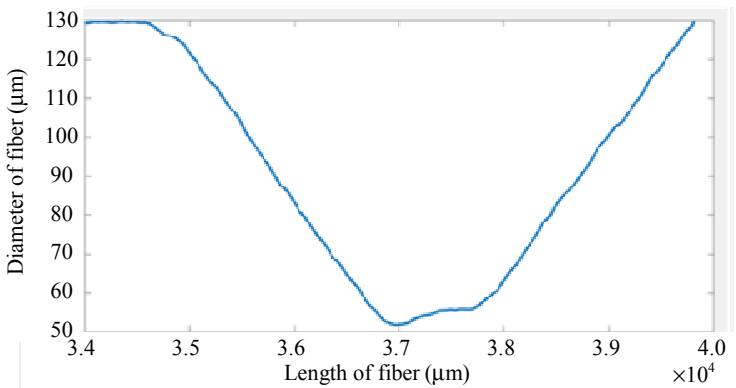

(c)

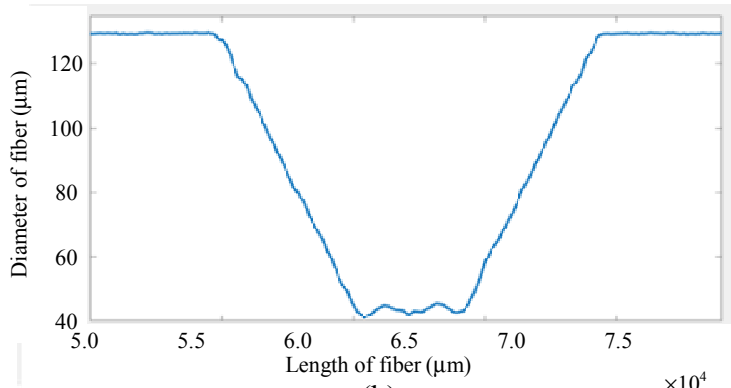

(b)

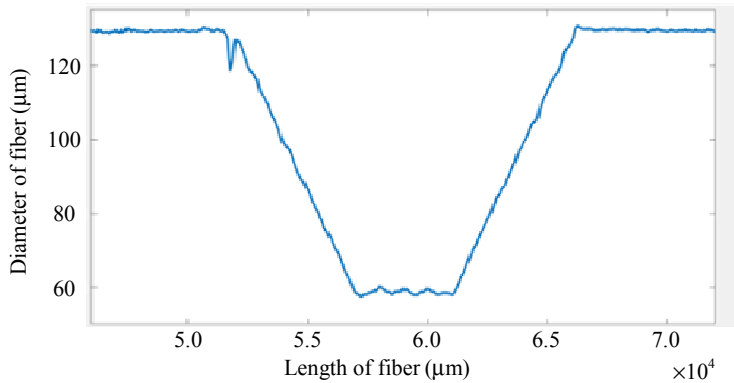

(d)

Fig. 5 Diameter analysis of fabricated tapered fiber structures plotted through MATLAB.

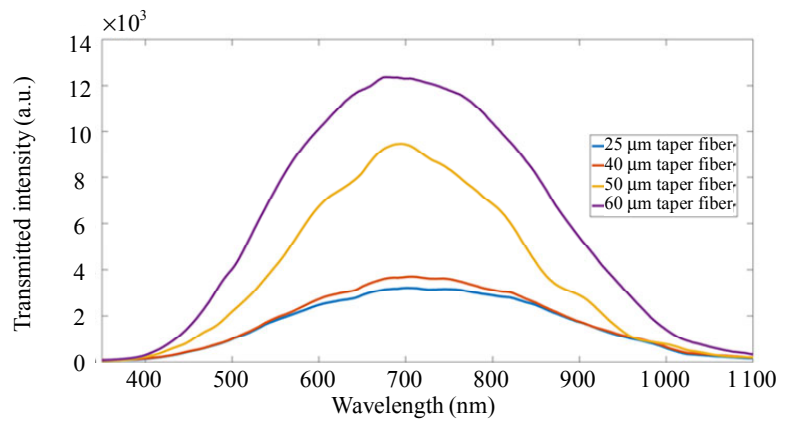

Fig. 6 Transmitted intensity spectra recorded through a different diameter of tapered fiber structures.

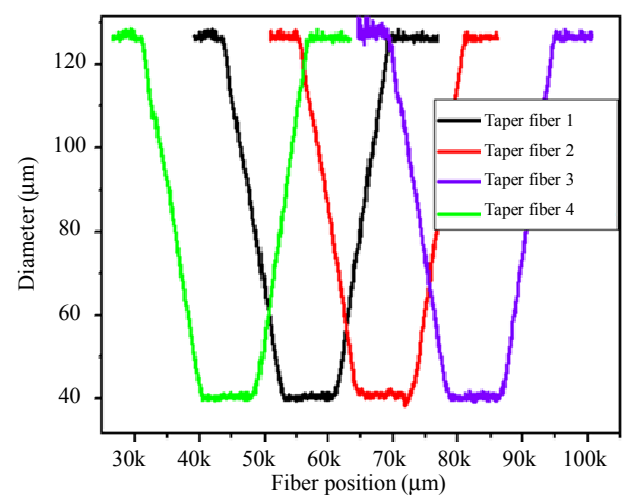

(a)

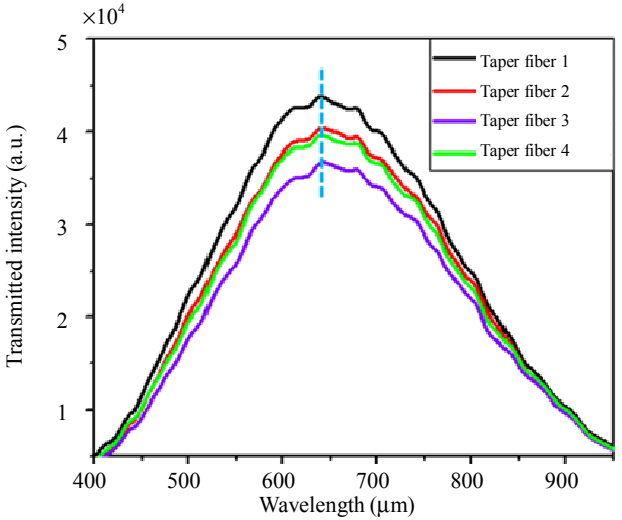

(b)

Fig. 7 Homogeneity analysis of the fabricated tapered fiber structures: (a) diameter analysis and (b) transmitted intensity.

\subsection{Characterization of nanoparticles}

The primary confirmation of NPs is done by measuring their absorbance peak wavelengths through a UV-vis spectrophotometer. To measure the absorbance spectrum, $2 \mathrm{ml}$ (1:1 AuNPs: DI water) of the AuNPs solution is poured in a quartz cuvette that 
has a path length of $1 \mathrm{~cm}$. The quartz cuvette is used because of its property of absorbing UV radiation. The absorbance peak wavelength for the AuNPs appears at $519 \mathrm{~nm}$, as shown in Fig. 8(a). Thereafter, the distribution of AuNPs inside the solution is observed by capturing an image through HR-TEM, as shown in Fig. 8(b). The average size of synthesized AuNPs is around $(11 \pm 0.2) \mathrm{nm}$ as can be seen from the histogram presented in Fig. 8(c), which is measured by using ImageJ software.

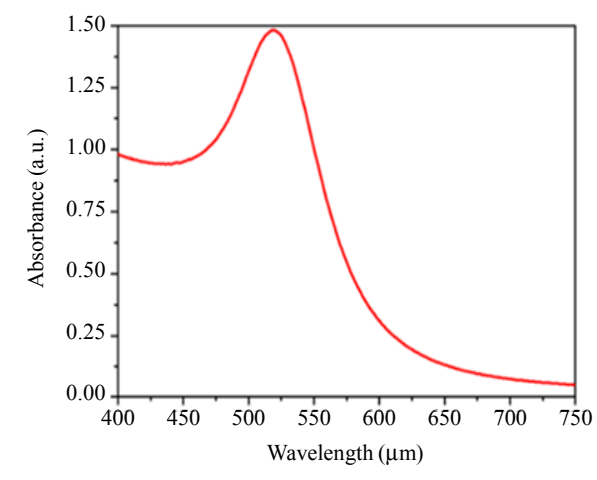

(a)

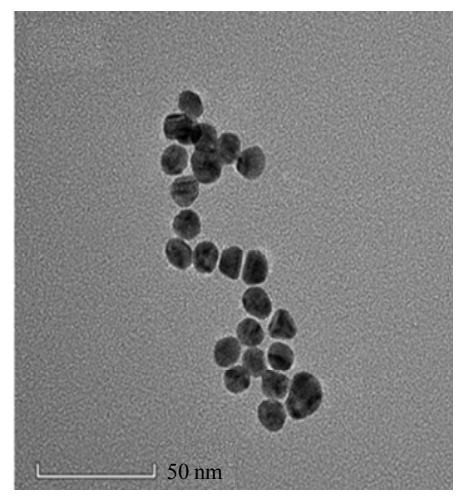

(b)

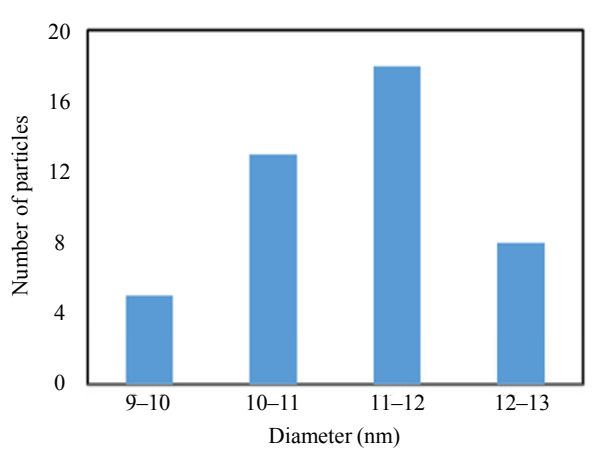

(c)

Fig. 8 Characterizations of gold nanoparticles: (a) absorbance spectrum, (b) TEM image, and (c) histogram showing the average size of gold nanoparticles is $11 \pm 0.2 \mathrm{~nm}$.

Similarly, $10 \mu \mathrm{l}$ of the ZnO-NPs solution (20\% wt.) is dissolved in $1.99 \mathrm{ml}$ of DI water and used for measuring the absorbance peak wavelength that appears at $370 \mathrm{~nm}$ as shown in Fig. 9(a). The peak wavelength at $370 \mathrm{~nm}$ corresponds to the formation of the $\mathrm{ZnO}-\mathrm{NPs}$ of size, less than $50 \mathrm{~nm}$. Thereafter, the distribution of NPs inside the solution is observed from the TEM image as presented in

Fig. 9(b). The deposition of the ZnO-NPs layer over the sensing region provides a larger surface area and a better bonding strength [36]. The surface morphology of $\mathrm{ZnO}-\mathrm{NPs}$ is done by capturing an AFM image as shown in Fig. 9(c). The high roughness can be observed from the result that is quite helpful for binding the biomolecules and increasing the specificity.

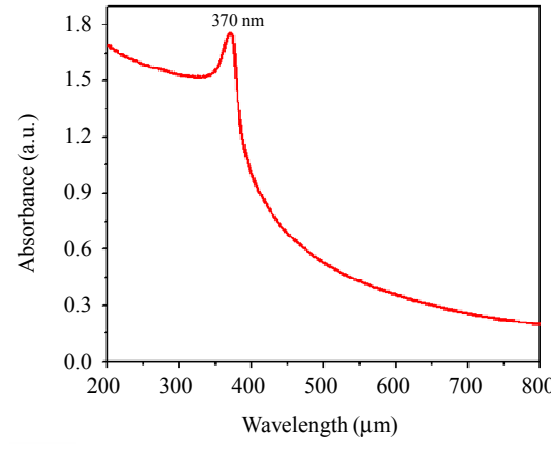

(a)

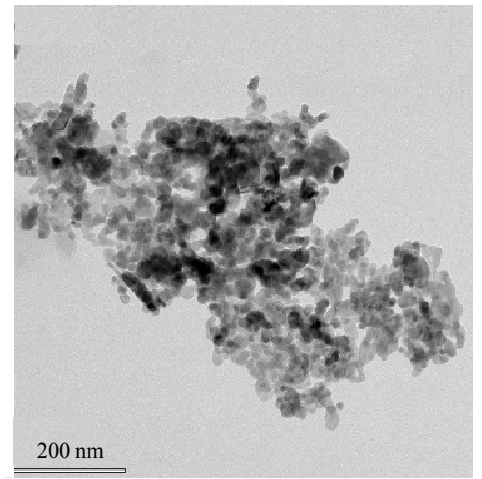

(b)

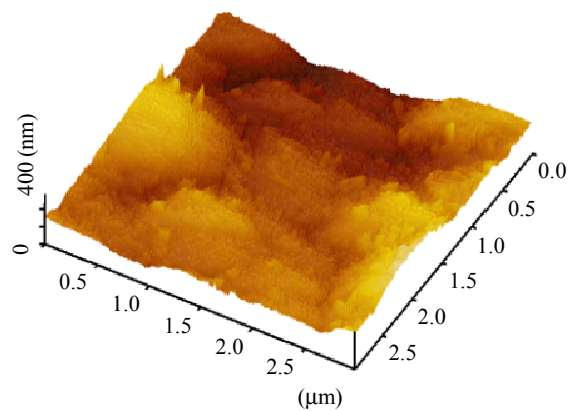

(c)

Fig. 9 Characterizations of zinc oxide nanoparticles: (a) absorbance spectrum, (b) TEM image, and (c) AFM image.

\subsection{Characterization of nanoparticles coated fiber probes}

To excite the LSPR, the immobilization of AuNPs over the fiber surface should be uniform throughout the sensing region. Therefore, to observe and confirm the NPs-coating over the tapered region, fiber probes are characterized by capturing the images through SEM. For Probe I, an image is 
captured at the $28 \mathrm{X}$ magnification to observe the coating of AuNPs over the sensing region, as shown in Fig. 10(a). The distribution of NPs over the sensing region is observed by taking an image at a higher magnification of 50KX (times), as shown in Fig. 10(b). From the result, one can easily observe

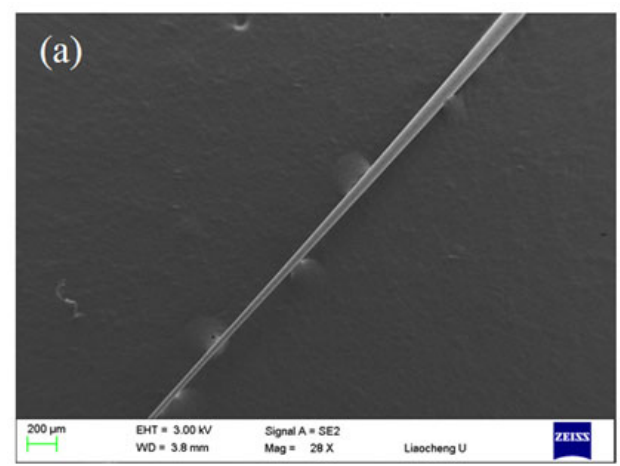

the uniform distribution of AuNPs over the fiber surface. Similarly, for Probe II, an image is captured at the magnification of $50 \mathrm{KX}$ to observe the distribution of AuNPs and ZnO-NPs over the sensing region as shown in Fig. 11.

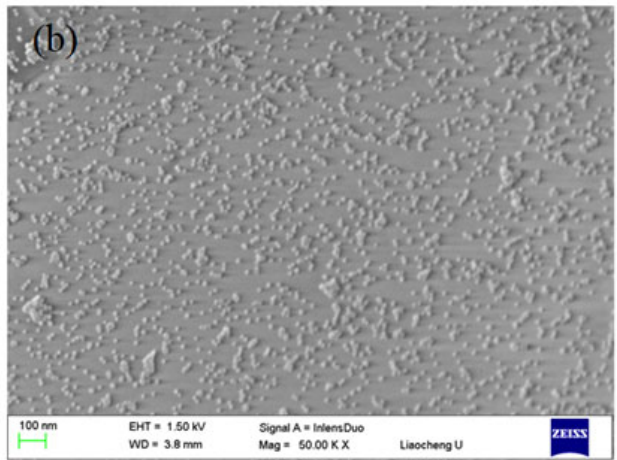

Fig. 10 SEM images of the nanoparticles-immobilized sensor probe: (a) SEM image of Probe I and (b) gold nanoparticles at the surface of the tapered fiber structure.

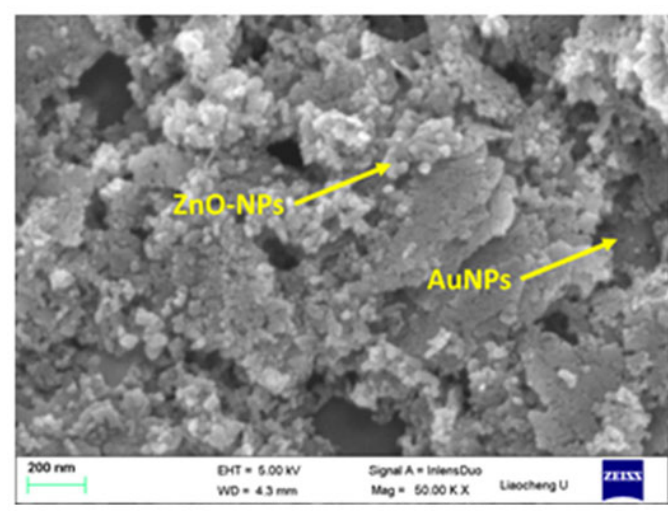

Fig. 11 SEM image of Probe II, gold, and zinc oxide nanoparticles over the surface of the taper fiber structure.

\subsection{LSPR sensing by using the developed probes}

The developed sensor probes are used to sense different concentrations of AA solutions from $0.5 \mu \mathrm{M}$ to $1 \mathrm{mM}$. This range completely covers the lowest and highest concentrations of AA found in human bodies. The testing of all the concentrations is done in the ascending order. Before sensing the first concentration solution of AA, the probe is rinsed with a base solution and dried. After drying the sensor head, the respective transmitted intensity and wavelength are recorded. Then, the probe is rinsed again with a base solution and dried at room temperature before measuring the other concentration solutions of AA. Likewise, the sensing of all the samples is done with both the developed sensor probes, and the attained results are presented in terms of transmitted intensity spectra and linearity range curves. Initially, the $0.5 \mu \mathrm{M}$ concentration of the AA solution is added in the vicinity of the sensor head and the respective LSPR spectrum is recorded. All the solutions are tested three times and their averaged values of LSPR spectra are plotted. For Probe I, LSPR spectra are plotted in Fig. 12(a). As can be seen from the results, the transmitted intensity decreases as the concentration of the AA solution increases. In contrast, the peak resonance wavelength shows the opposite response such that it increases on increasing the concentration of AA solutions. To plot the transmitted intensity curves, the experiment is repeated three times with three different sensor probes. Afterwards, the overall spectra are plotted by normalizing the plots from each experiment. The unusual behavior might be due to different readings obtained from different experiments. The variation in the transmitted intensity curve and peak 
resonance wavelength is due to the change in imaginary and real parts of the refractive index of the sensing layer. This refractive index is varying with the addition of different concentration solutions of AA. The variation in the transmitted intensity and peak resonance wavelength is also due to the $\mathrm{AOx}$ enzyme. The immobilization is very specific towards AA solutions and gets oxidized. Due to the oxidation reaction, the products vary the refractive index in the vicinity of the sensor head that further alters the properties of EWs. These EWs further interact with the signal propagating within the probe and causes a shift in its amplitude and wavelength. But, the overall results of the experiment such as the linearity range and sensitivity are concluded from the linearity range curve. From the linearity range

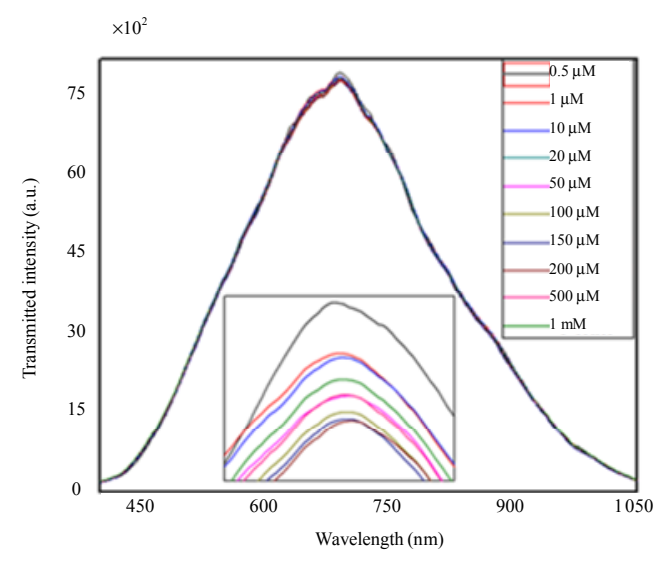

(a) curve, one can easily observe an increase in the wavelength with respect to the concentration of AA solutions. This inverse behavior is due to the reduction in the imaginary part of RI of the sensing layer. The response of Probe I shows a good linearity with the autocorrelation fitting of $99.24 \%$ for the concentrations ranges from $1 \mu \mathrm{M}$ to $200 \mu \mathrm{M}$, as shown in Fig. 12(b). Similarly, the performance evaluation of Probe II is done by testing all the concentrations and the averaged LSPR spectra are plotted as shown in Fig. 13(a). This response is due to the production of reaction byproducts that can change the effective RI of the sensing layer [37]. In presented results, the readings of higher concentrations are skipped because of non-linearity attained in the results.

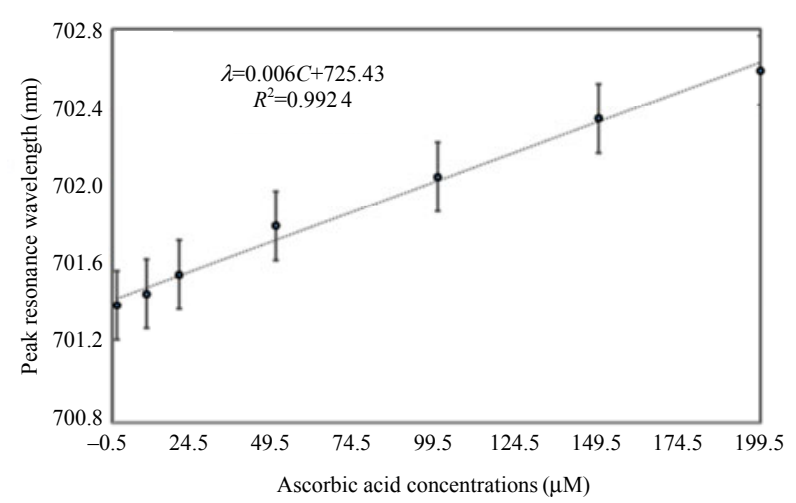

(b)

Fig. 12 LSPR sensing of Probe I: (a) transmitted intensity spectra and (b) linearity response of sensor.

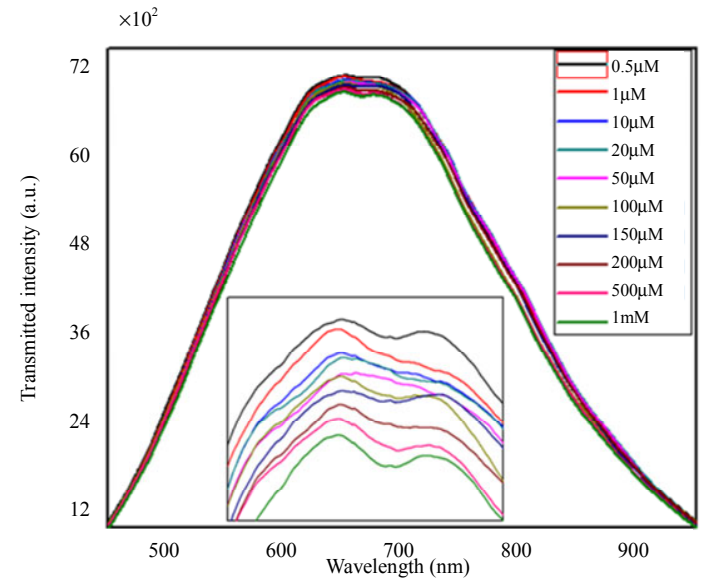

(a)

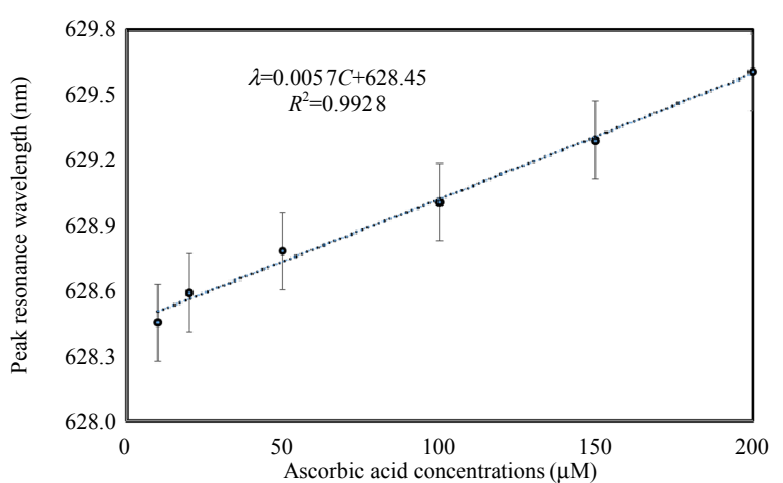

(b)

Fig. 13 LSPR sensing of Probe II: (a) transmitted intensity spectra and (b) linearity response of the sensor. 
For higher concentrations of the AA solutions, the peak resonance wavelength is saturated which is due to the filling of available binding sites over the sensing layer. It has been observed from Fig. 13(b) that the peak resonance wavelength is directly proportional to the AA concentrations. This redshift response is due to an increase in RI of the real part of the sensing layer. The response of Probe II also shows a good linearity in the concentration range between $10 \mu \mathrm{M}$ and $200 \mu \mathrm{M}$. The linearity response of sensor probes is attained with the autocorrelation curve fitting of $99.28 \%$.

The limit of detection $(L o D)[L o D=(3 \times$ standard deviation/sensitivity)] is a very crucial parameter for evaluating the performance of a sensor. It can be evaluated on the basis of the calibration curve by considering three times of the standard deviation of the blank measurement. If a few concentrations of the samples have been considered, then $L o D$ can be calculated from the calibration curve through inverse fitting function to a particular point such as the mean value of the blank sample plus three times the maximum standard deviation obtained among all the experimental points [38]. For Probe I, the attained value of standard deviation $(S D)$ is 0.0253 . Therefore, the attained $L o D$ of Probe I for sensing the AA concentrations from $1 \mu \mathrm{M}$ to $200 \mu \mathrm{M}$ is $12.65 \mu \mathrm{M}$. With this $L o D$, the developed probe can sense the concentration of AA found in human bodies, i.e., $40 \mu \mathrm{M}$ to $120 \mu \mathrm{M}$. The sensitivity of Probe I for the concentrations ranges from $1 \mu \mathrm{M}$ to $200 \mu \mathrm{M}$ is $6 \mathrm{~nm} / \mathrm{mM}$, which can be obtained from the slope of the linearity curve. In a similar manner, the evaluated $L o D$ for Probe II is $25.78 \mu \mathrm{M}$ for the concentration ranges from $10 \mu \mathrm{M}$ to $200 \mu \mathrm{M}$. The sensitivity response of Probe II is around $5.7 \mathrm{~nm} / \mathrm{mM}$ as obtained from the curve fitting of the auto-correlation curve.

\subsection{Performance analysis of the developed sensing probes}

To observe the solubility of AA molecules, a $\mathrm{pH}$ test is also done by dissolving its lowest and highest concentrations in different $\mathrm{pH}$ values solutions. Further, the performance evaluation of the developed sensor probes is also done in terms of stability, reproducibility, and repeatability.

\subsection{1 $\mathrm{pH}$ test}

This test is done to observe the solubility of AA molecules in different $\mathrm{pH}$ value solutions. The solubility is tested by dissolving the lowest and highest tested concentrations of AA in five different solutions having a pH value of 5 (ethanol), 7.4 (PBS), 8, 11, and 14. The basic nature solutions are prepared by adjusting the $\mathrm{pH}$ of DI water by using $\mathrm{NaOH}$. Initially, the lower concentration of one solution is added in the vicinity of the sensor head and the respective spectrum is measured. Then, the probe is rinsed with a blank solution with the same $\mathrm{pH}$ and dried at room temperature. Thereafter, the higher concentration of the same solution is tested. Likewise, all the solutions are tested and the corresponding LSPR spectra are stored. Afterwards, the difference in the peak resonance wavelength is evaluated from higher to lower concentration and plotted with respect to the $\mathrm{pH}$ value of the solution as shown in Fig. 14. From the results, it is ascertained that the maximum difference of the peak wavelength is observed when the AA solution is prepared in the PBS solution ( $\mathrm{pH}$ 7.4). Hence, the PBS is used to prepare all the AA solutions.

\subsubsection{Stability test}

The stability test is performed to observe the response of the developed sensor probes against the number of measurements. To perform the test, the blank solution (PBS) is tested 15 times through the same sensing probe and the respective LSPR spectra are recorded. Thereafter, the peak wavelength is evaluated from each measurement and plotted with respect to the measurements. For Probe I, the obtained results for stability tests are presented in Fig. 15(a). From the results, it can be observed that 
the peak wavelength is the same almost for all the measurements with an $S D$ of 0.025 . Whereas, in LSPR spectra plotted for Probe I in Fig. 12(a), the attained intensity counts for PBS measurement are lower. This is because the PBS measurement is done by another sensor probe, whereas the LSPR spectra are averaged of three different measurements. In reality, the imaginary part of $\mathrm{RI}$ reduces on increasing the concentration of AA which can be easily observed from the spectra presented in inset of Fig. 12(a). In a similar manner, the results obtained for Probe II are presented in Fig. 15(b). The response of Probe II is also very linear that can be observed from the evaluated $S D$ of 0.049 . The attained stability shows that the developed fiber sensor probes can be used to measure at least 15 concentrations with the attained values of $S D$.

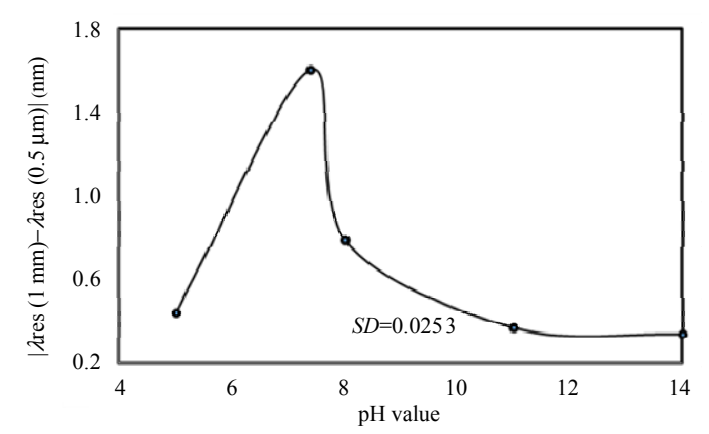

Fig. $14 \mathrm{pH}$ test to determine the solubility of AA molecules.

\subsubsection{Reproducibility}

The authentication of the used protocols to develop the sensor probes can be done by performing the reproducibility test. To perform the test, the $1 \mu \mathrm{M}$ concentration of the AA solution is tested by using three different sensor probes and the respective LSPR spectra are recorded. Then, the result is analyzed on the basis of variation in the peak resonance wavelength for all the measurements. For Probe I, the obtained results are presented in Fig. 16(a). From the results, one can easily observe that the peak wavelengths of all the measurements are almost similar that ensures the good reproducibility of the used protocols. Similarly, for Probe II, the $10 \mu \mathrm{M}$ concentration of the AA solution is tested through three different sensor probes and the obtained results are presented in Fig. 16(b). In presented results, one can observe the overlapping of curves which is due to the similar concentration of the AA solution that proves the good reproducibility of the developed sensor probes. In a comparison of Fig. 15, the curves presented in Fig. 16 are a bit different because in former one, the complete curve are presented, whereas, in the later one, curves are zoomed to a particular point to clearly observe the spectra.

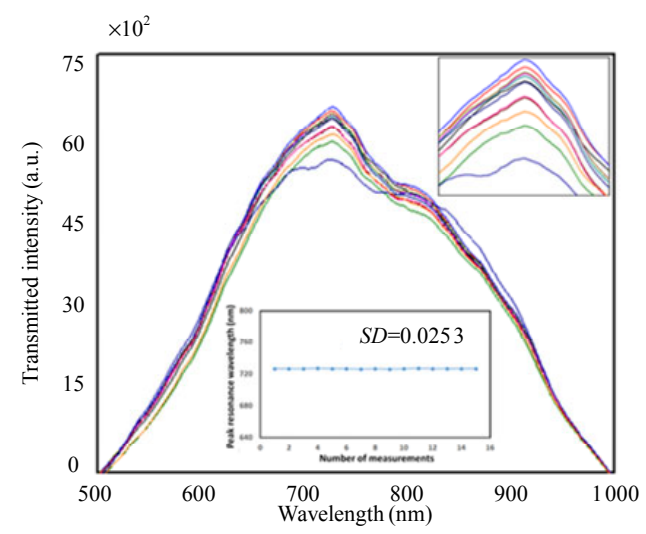

(a)

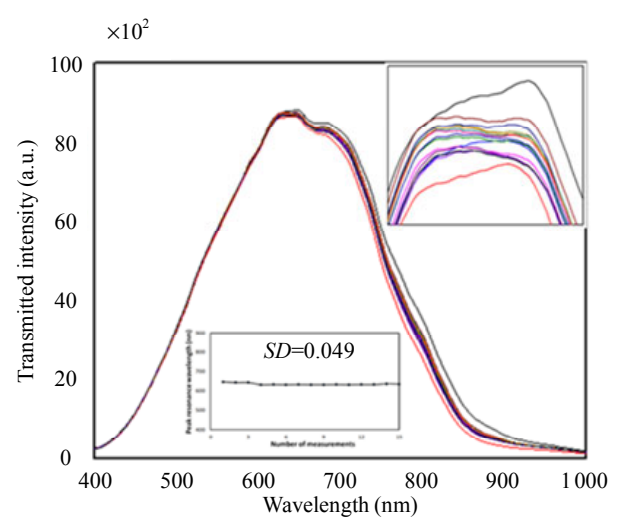

(b)

Fig. 15 Stability test: (a) Probe I and (b) Probe II.

\subsubsection{Repeatability test}

It is another important aspect of a sensor to observe its responses for similar solutions. To perform the repeatability test, two different concentrations of AA solutions such as $0.5 \mu \mathrm{M}$ and $150 \mu \mathrm{M}$ are tested twice through the same sensor probes. Firstly, the $0.5 \mu \mathrm{M}$ concentration solution is added near to the sensor head and the respective 
LSPR spectrum is recorded. Then, the probe is rinsed with the PBS solution and dried at room temperature. Then, the same concentration of the solution is tested through the same sensing probe.

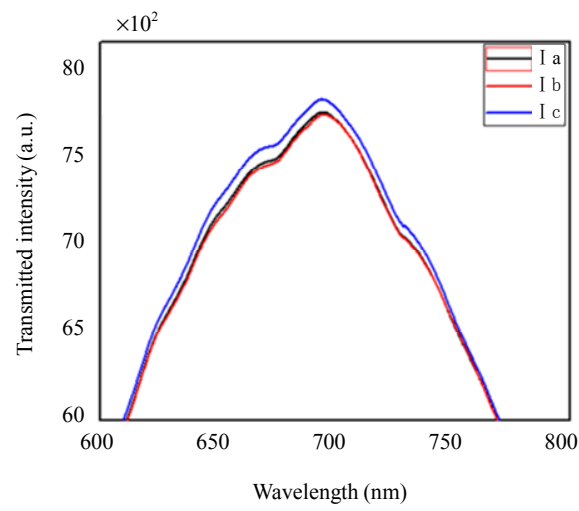

(a)

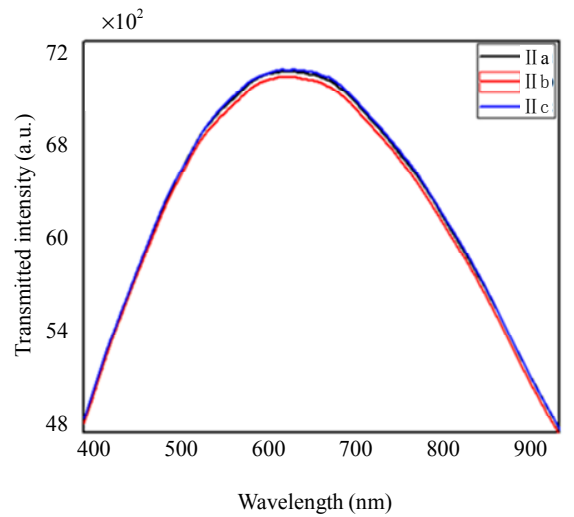

(b)

Fig. 16 Reproducibility test: (a) Probe I and (b) Probe II.

Similarly, the solution of the $150 \mu \mathrm{M}$ concentration is tested by recording the respective LSPR spectra. The change in the amplitude of the transmitted intensity spectrum is due to the variation in the imaginary part of the refractive index of the sensing layer. Moreover, in this work, we are claiming the results on the basis of the peak resonance wavelength which is similar for the tested concentration of the AA solutions. The obtained results for Probes I and II are presented in Figs. 17(a) and $17(\mathrm{~b})$, respectively. From the results, it is ascertained that the peak wavelength for the same concentration is not changed, which ensures the fine repeatability of the developed probes.

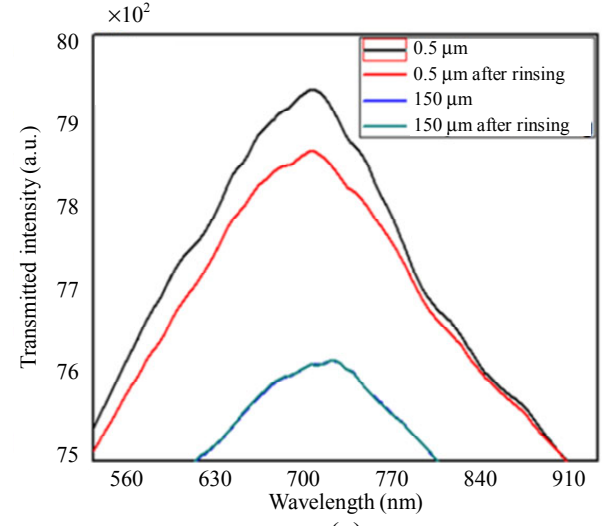

(a)

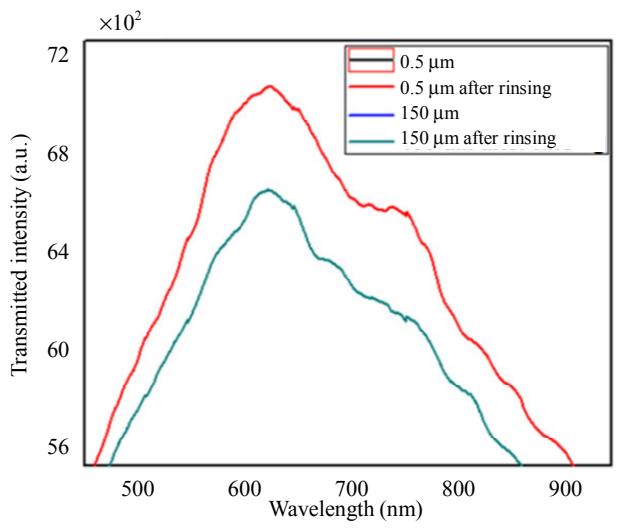

(b)

Fig. 17 Repeatability test: (a) Probe I and (b) Probe II.

\subsection{Selectivity of the sensor probe}

Selectivity is also a very important aspect of biosensors. The proposed sensor is enzymatic in nature that is used to detect the AA in human bodies. Several biomolecules such as glucose, cholesterol, D-galactose, dopamine, and urea are found in the human bodies which are used to analyze the specificity of the developed sensor probes. Figures 18(a) and 18(b) show the results of the selectivity test of Probes I and II, respectively. To test these biomolecules, the solutions of lower and higher concentrations as per the linear range are prepared by using a PBS solution. Initially, a solution of the lower concentration is dropped on the sensor head and the LSPR spectrum is recorded. Thereafter, the sensor probe is cleaned with the PBS solution and dried at room temperature. After 20 minutes, a solution of the $200 \mu \mathrm{M}$ concentration of biomolecules is tested with the same sensing probe. 
Likewise, the experiments are repeated for all the mentioned biomolecules. Then, the wavelength is extracted from all the measurements and plotted by taking the difference between higher and lower concentrations of the solution. From the results, it is concluded that the developed sensor probes have very high selectivity towards AA. The higher selective response of the developed probes is observed due to the oxidation of AA in the presence of AOx enzyme with oxygen, as shown in Fig. 19.

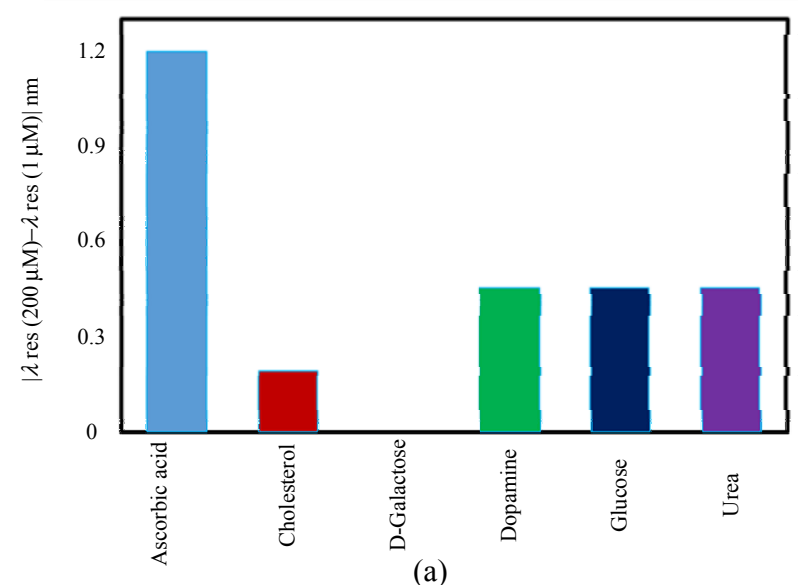

Fig. 18 Selectivity test: (a) Probe I and (b) Probe II.

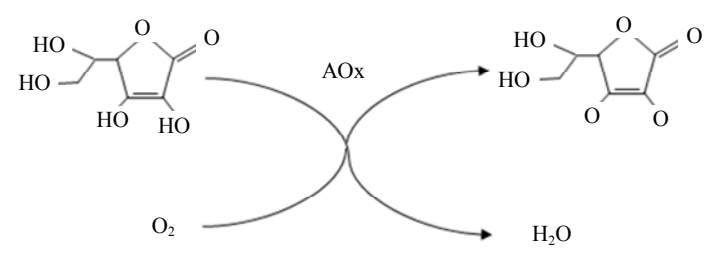

Fig. 19 Reduction of ascorbic acid in the presence of AOx.

\subsection{Performance study of the proposed sensor}

The performance comparison of the developed sensor probes has been done with the existing works in terms of the linearity range, detection limit, and sensitivity.

The attained results of the developed sensor probes are compared with some of the existing studies and presented in Table 1. For instance, two different studies are carried out while completely covering the available range of AA presents in human bodies. The claimed detection limit of sensor structures is also good, but there is no discussion
From the results, one can observe that the glucose shows a higher peak in selectivity tests and its concentration in human bodies is also up to the $\mathrm{mM}$ range. Instead of having ascorbate oxidase enzyme over the sensing region, the response from other biomolecules is only due to the carbon chain structures. But in comparison with AA, the response of the developed sensor probe is very less for other biomolecules which is due to the presence of $\mathrm{AOx}$ enzyme over the sensing region.

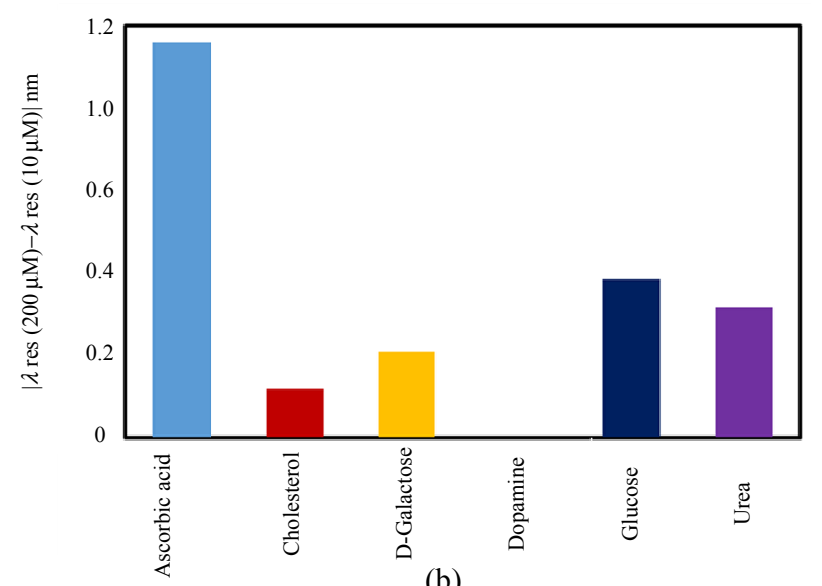

(b)

about the sensitivity of sensor probes [8, 39]. Similarly, two different sensor structures based on colorimetric schemes are also claiming the better detection limit to detect the presence of AA. But, the tested range of $\mathrm{AA}$ is not covering its complete range which is available in human bodies and there is no discussion about the attained sensitivity of sensor probes [39, 40]. In another work, gold nanoclusters are used to sense the presence of AA by using the fluorescence technique [41]. The tested range of AA solutions is not covering its concentration available in human bodies and even there is no discussion about the attained sensitivity.

In our work, we have tested the AA in a very wide concentration from $0.5 \mu \mathrm{M}$ to $1 \mathrm{mM}$, and for the range of $40 \mu \mathrm{M}$ to $200 \mu \mathrm{M}$, the sensor response is very fine in terms of its linearity range $(99.24 \%$ for Probe I and $99.28 \%$ for Probe II) and sensitivity $(6 \mathrm{~nm} / \mathrm{mM}$ for Probe I and $5.7 \mathrm{~nm} / \mathrm{mM}$ for Probe II). 
Whereas, the sensor probes developed in the present work are able to cover the complete range of AA found in human bodies with very high sensitivity, which is better than those of the compared works.

Table 1 Performance study of the proposed sensors.

\begin{tabular}{|c|c|c|c|c|c|}
\hline Material used & $\begin{array}{c}\text { Mechanism } \\
\text { used }\end{array}$ & Linear range & $\begin{array}{c}\text { Detection } \\
\text { limit }\end{array}$ & Sensitivity & Ref. \\
\hline $\begin{array}{l}\text { Carbon } \\
\text { electrode }\end{array}$ & Electrochemical & $\begin{array}{l}0.7 \mu \mathrm{M}- \\
11.5 \mathrm{mM}\end{array}$ & $0.24 \mu \mathrm{M}$ & n.r. & [39] \\
\hline $\begin{array}{c}\text { Manganese } \\
\text { (IV) oxide } \\
\left(\mathrm{MnO}_{2}\right) \mathrm{NPs}\end{array}$ & Electrochemica & $\begin{array}{c}2.64 \mu \mathrm{M}- \\
1.5 \mathrm{mM}\end{array}$ & $0.8 \mu \mathrm{M}$ & n.r. & [8] \\
\hline $\begin{array}{c}\text { Reduced } \\
\text { graphene } \\
\text { oxide }\end{array}$ & Colorimetric & $0.8 \mu \mathrm{M}-60 \mu \mathrm{M}$ & $0.15 \mu \mathrm{M}$ & n.r. & {$[40]$} \\
\hline $\begin{array}{c}\text { Gold } \\
\text { nanoclusters }\end{array}$ & Fluorescence & $0.1 \mu \mathrm{M}-10 \mu \mathrm{M}$ & $22 \mathrm{nM}$ & n.r. & [41] \\
\hline $\begin{array}{l}\text { 3,3/,5,5/-tetra } \\
\text { methylbenzidine }\end{array}$ & Colorimetric & $1 \mu \mathrm{M}-70 \mu \mathrm{M}$ & $0.58 \mu \mathrm{M}$ & n.r. & {$[42]$} \\
\hline \multirow{2}{*}{ Probe I } & \multirow{2}{*}{ LSPR } & $1 \mu \mathrm{M}-200 \mu \mathrm{M}$ & $12.56 \mu \mathrm{M}$ & $6 \mathrm{~nm} / \mathrm{mM}$ & \multirow{2}{*}{$\begin{array}{r}\text { This } \\
\text { work }\end{array}$} \\
\hline & & $10 \mu \mathrm{M}-200 \mu \mathrm{M}$ & $25.78 \mu \mathrm{M}$ & $5.7 \mathrm{~nm} / \mathrm{mM}$ & \\
\hline
\end{tabular}

n.r. - not reported.

\section{Conclusions}

In this paper, a waist diameter of $40 \mu \mathrm{m}$ of the tapered optical fiber structure is fabricated by using the plasma technique. To enrich the probes with the applications of the LSPR phenomenon and increase their biocompatibility, AuNPs and ZnO-NPs are immobilized over the sensing region of probes. On the basis of two different configurations of NPs, two kinds of probes are developed. In the first probe, the detection of AA is done by using the AuNPs over the sensing region. Whereas, in another probe, the layer of AuNPs is further immobilized with a layer of ZnO-NPs. The authentication of probes is done by testing different concentrations of the AA solution ranges from $0.5 \mu \mathrm{M}$ to $1 \mathrm{mM}$. The validation of the AuNPs and ZnO-NPs solution is done by taking the pictures through the high-resolution TEM. Thereafter, the confirmation of NPs immobilization over the sensing region of the developed probes is done by taking the image through the SEM. Additionally, the specificity of the probes is enhanced by functionalizing them with AOx enzyme which is oxidized only in the presence of AA. The sensing response of the developed probes is ascertained by observing the shift in the peak resonance wavelength with respect to different concentrations of the AA solutions. Further, the authentication of the probes is done by analyzing their repeatability, reproducibility, and selectivity. The attained values for curve fitting and $L o D$ of Probes I and II are $99.24 \%$ and $99.28 \%$, and $12.56 \mu \mathrm{M}$ and $25.78 \mu \mathrm{M}$, respectively. Whereas, the attained values for the sensitivity of Probes I and II for the linearity range of $1 \mu \mathrm{M}-200 \mu \mathrm{M}$ and $10 \mu \mathrm{M}-200 \mu \mathrm{M}$ are $6 \mathrm{~nm} / \mathrm{mM}$ and $5.7 \mathrm{~nm} / \mathrm{mM}$, respectively. Therefore, from the attained results, it is ascertained that the response of Probe I is better in terms of its linearity range and $L o D$.

\section{Acknowledgment}

This work was supported by the National Key Research \& Development Program of China (Grant No. 2016YFB0402105), the Belt and Road Special Project approved by Shandong Province for the Introduction of Foreign Experts in 2018, Double-Hundred Talent Plan of Shandong Province, Liaocheng University, China (Grant Nos. 31805180301 and 31805180326), and Science and Engineering Research Board (SERB), India (Grant No. TAR/2018/000051).

Open Access This article is distributed under the terms of the Creative Commons Attribution 4.0 International License (http://creativecommons.org/licenses/by/4.0/), which permits unrestricted use, distribution, and reproduction in any medium, provided you give appropriate credit to the original author(s) and the source, provide a link to the Creative Commons license, and indicate if changes were made.

\section{References}

[1] J. F. Turrens, "Mitochondrial formation of reactive oxygen species," The Journal of Physiology, 2003, 552(2): 335-344.

[2] L. Jiang, K. Yang, J. Tian, Q. Guan, N. Yao, N. Cao, 
et al., "Efficacy of antioxidant vitamins and selenium supplement in prostate cancer prevention: a meta-analysis of randomized controlled trials," Nutrition and Cancer, 2010, 62(6): 719-727.

[3] M. Levine, S. C. Rumsey, R. Daruwala, J. B. Park, and Y. Wang, "Criteria and recommendations for vitamin C intake," The Journal of the American Medical Association, 1999, 281(15): 1415-1423.

[4] D. T. Alexandrescu, C. A. Dasanu, and C. L. Kauffman, "Acute scurvy during treatment with interleukin-2," Clinical and Experimental Dermatology: Clinical Dermatology, 2009, 34(7): 811-814.

[5] M. T. Heafield, S. Fearn, G. B. Steventon, R. H. Waring, A. C. Williams, and S. G. Sturman, "Plasma cysteine and sulphate levels in patients with motor neurone, Parkinson's and Alzheimer's disease," Neuroscience Letters, 1990, 110(1-2): 216-220.

[6] S. J. Padayatty, A. Katz, Y. Wang, P. Eck, O. Kwon, J. Lee, et al., "Vitamin $\mathrm{C}$ as an antioxidant: evaluation of its role in disease prevention," Journal of the American College of Nutrition, 2003, 22(1): 18-35.

[7] K. Matsumoto, K. Yamada, and Y. Osajima, "Ascorbate electrode for determination of L-ascorbic acid in food," Analytical Chemistry, 1981, 53(13): 1974-1979.

[8] S. Çevik, O. Akpolat, and Ü. Anik, "Ascorbic acid detection with $\mathrm{MnO}_{2}$-modified GCPE," Food Analytical Methods, 2016, 9(2): 500-504.

[9] J. Liu, Y. Chen, W. Wang, J. Feng, M. Liang, S. Ma, et al., "'Switch-On' fluorescent sensing of ascorbic acid in food samples based on carbon quantum dots- $\mathrm{MnO}_{2}$ probe," Journal of Agricultural and Food Chemistry, 2016, 64(1): 371-380.

[10] W. J. Ni, D. Shan, R. H. Zhu, S. Y. Deng, S. Cosnier, and X. J. Zhang, "Dumbbell-shaped carbon quantum dots/AuNCs nanohybrid as an efficient ratiometric fluorescent probe for sensing cadmium (II) ions and 1-ascorbic acid," Carbon, 2016, 96: 1034-1042.

[11] J. Chen, J. Ge, L. Zhang, Z. Li, J. Li, Y. Sun, et al., "Reduced graphene oxide nanosheets functionalized with poly (styrene sulfonate) as a peroxidase mimetic in a colorimetric assay for ascorbic acid," Microchimica Acta, 2016, 183(6): 1847-1853.

[12] D. R. S. Lima, M. Cossenza, C. G. Garcia, C. C. Portugal, F. F. de C. Marques, R. Paes-de-Carvalho, et al., "Determination of ascorbic acid in the retina during chicken embryo development using high performance liquid chromatography and UV detection," Analytical Methods, 2016, 8(27): 5441-5447.

[13] S. Zang, S. Tian, J. Jiang, D. Han, X. Yu, K. Wang, et al., "Determination of antioxidant capacity of diverse fruits by electron spin resonance (ESR) and UV-vis spectrometries," Food Chemistry, 2017, 221: 1221-1225

[14] I. Biran, X. Yu, and D. R. Walt, Optical biosensors:
Chapter 1 - optrode-based fiber optic biosensors (bio-optrode) (Second edition). Amsterdam: Elsevier, 2008: 3-82.

[15] A. B. Socorro, E. Santamaría, J. F. Irigoyen, I. D. Villar, J. M. Corres, F. J. Arregui, et al., "Fiber-optic immunosensor based on an etched SMS structure," IEEE Journal of Selected Topics in Quantum Electronics, 2016, 23(2): 314-321.

[16] S. Kumar, B. K. Kaushik, R. Singh, N. K. Chen, Q. S. Yang, X. Zhang, et al., "LSPR-based cholesterol biosensor using a tapered optical fiber structure," Biomedical Optics Express, 2019, 10(5): 2150-2160.

[17] K. Li, G. Liu, Y. Wu, P. Hao, W. Zhou, and Z. Zhang, "Gold nanoparticle amplified optical microfiber evanescent wave absorption biosensor for cancer biomarker detection in serum," Talanta, 2014, 120: 419-424.

[18] Y. Li, H. Ma, L. Gan, Q. Liu, Z. Yan, D. Liu, et al., "Immobilized optical fiber microprobe for selective and high sensitive glucose detection," Sensors and Actuators B: Chemical, 2018, 255: 3004-3010.

[19] N. M. Y. Zhang, M. Qi, Z. Wang, Z. Wang, M. Chen, $\mathrm{K}$. Li, et al., "One-step synthesis of cyclodextrin-capped gold nanoparticles for ultra-sensitive and highly-integrated plasmonic biosensors," Sensors and Actuators B: Chemical, 2019, 286: 429-436.

[20] G. Zheng, Y. Liu, L. Shi, R. Zhao, and T. Wang, "Reflective index sensors based on tilted fiber Bragg grating inscribed in the thin-core fiber," in 201615 th International Conference on Optical Communications and Networks (ICOCN), China, Sept. 24-27, 2016, pp. 1-3.

[21] M. Batumalay, S. W. Harun, F. Ahmad, R. M. Nor, N. R. Zulkepely, and H. Ahmad, "Tapered plastic optical fiber coated with graphene for uric acid detection," IEEE Sensors Journal, 2014, 14(5): 1704-1709.

[22] L. Singh, G. Zhu, R. Singh, B. Zhang, W. Wang, B. K. Kaushik, et al., "Gold nanoparticles and uricase functionalized tapered fiber sensor for uric acid detection," IEEE Sensors Journal, 2019, 20(1): 219-226.

[23] C. T. Lee, M. L. Wu, L. G. Sheu, P. L. Fan, and J. M. Hsu, "Design and analysis of completely adiabatic tapered waveguides by conformal mapping," Journal of Lightwave Technology, 1997, 15(2): 403-410.

[24] J. L. Hammond, N. Bhalla, S. D. Rafiee, and P. Estrela, "Localized surface plasmon resonance as a biosensing platform for developing countries," Biosensors (Basel), 2014, 4(2): 172-188.

[25] A. K. Sharma, J. Gupta, and I. Sharma, "Fiber optic evanescent wave absorption-based sensors: a detailed review of advancements in the last decade (2007-18)," Optik, 2019, 183: 1008-1025. 
[26] X. Guo, "Surface plasmon resonance-based biosensor technique: a review," Journal of Biophotonics, 2012, 5(7): 483-501.

[27] J. Turkevich, P. C. Stevenson, and J. Hillier, "A study of the nucleation and growth processes in the synthesis of colloidal gold," Discussions of the Faraday Society, 1951, 11: 55-75.

[28]H. M. Kim, D. H. Jeong, H. Y. Lee, J. H. Park, and S. K. Lee, "Improved stability of gold nanoparticles on the optical fiber and their application to refractive index sensor based on localized surface plasmon resonance," Optics \& Laser Technology, 2019, 114: 171-178.

[29]I. Arghir, D. Spasic, B. E.Verlinden, F. Delport, and J. Lammertyn, "Improved surface plasmon resonance biosensing using silanized optical fibers," Sensors and Actuators B: Chemical, 2015, 216: 518-526.

[30] S. Aza, E. Sadeghi, R. Parvizi, A. Mazaheri, and M. Yousefi, "Sensitivity optimization of $\mathrm{ZnO}$ clad-modified optical fiber humidity sensor by means of tuning the optical fiber waist diameter," Optics \& Laser Technology, 2017, 90: 96-101.

[31]L. Coelho, D. Viegas, J. L. Santos, and J. D. Almeida, "Characterization of zinc oxide coated optical fiber long period gratings with improved refractive index sensing properties," Sensors and Actuators B: Chemical, 2016, 223: 45-51.

[32] F. Qu, H. Lu, M. Yang, and C. Deng, "Electrochemical immunosensor based on electron transfer mediated by graphene oxide initiated silver enhancement," Biosensors and Bioelectronics, 2011, 26(12): 4810-4814.

[33] N. EllinaAzmi, A. H. A. Rashid, J. Abdullah, N. A.Yusof, and H. Sidek, "Fluorescence biosensor based on encapsulated quantum dots/enzymes/sol-gel for non-invasive detection of uric acid," Journal of Luminescence, 2018, 202: 309-315.

[34] A. M. Shrivastav, S. K. Mishra, and B. D. Gupta, "Surface plasmon resonance-based fiber optic sensor for the detection of ascorbic acid utilizing molecularly imprinted polyaniline film," Plasmonics, 2015, 10(6): 1853-1861.

[35] G. K. Schwalfenberg, "The alkaline diet: is there evidence that an alkaline $\mathrm{pH}$ diet benefits health?,' Journal of Environmental and Public Health, 2012, 2012: 727630 .

[36] Z. Samavati, A. Samavati, A. F. Ismail, M. H. D. Othman, and M. A. Rahman, "Comprehensive investigation of evanescent wave optical fiber refractive index sensor coated with $\mathrm{ZnO}$ nanoparticles," Optical Fiber Technology, 2019, 52: 101976.

[37] R. Kant and B. D. Gupta, "Fiber-optic SPR based acetylcholine biosensor using enzyme functionalized $\mathrm{Ta}_{2} \mathrm{O}_{5}$ nanoflakes for alzheimer's disease diagnosis," Journal of Lightwave Technology, 2018, 36(18): 4018-4024.

[38] J. Villatoro and D. Monzon-Hernandez, "Low-cost optical fiber refractive-index sensor based on core diameter mismatch," Journal of Lightwave Technology, 2006, 24(3): 1409-1413.

[39] Y. Song, C. Gong, D. Su, Y. Shen, Y. Song, and L. Wang, "A novel ascorbic acid electrochemical sensor based on spherical MOF-5 arrayed on a three-dimensional porous carbon electrode," Analytical Methods, 2016, 8(10): 2290-2296.

[40] J. Chen, J. Ge, L. Zhang, Z. Li, J. Li, Y. Sun, et al., "Reduced graphene oxide nanosheets functionalized with poly (styrene sulfonate) as a peroxidase mimetic in a colorimetric assay for ascorbic acid," Microchimica Acta, 2016, 183(6): 1847-1853.

[41] H. Meng, D. Yang, Y. Tu, and J. Yan, "Turn-on fluorescence detection of ascorbic acid with gold nanolcusters," Talanta, 2017, 165: 346-350.

[42] C. Mu, H. Lu, J. Bao, and Q. Zhang, "Visual colorimetric 'turn-off' biosensor for ascorbic acid detection based on hypochlorite-3,3',5,5',-Tetramethylbenzidine system," Spectrochimica Acta Part A: Molecular and Biomolecular Spectroscopy, 2018, 201: 61-66. 